\title{
Glossopharyngeal Neuralgia: MVD vs GKS
}

\author{
Caique Chagas Cavuto ${ }^{1}$, Enrico Affonso Barletta ${ }^{2 *}$, Ranieri Henrique Moraes Lopes Gaspar ${ }^{3}$, João Flávio \\ Mattos Araújo ${ }^{4}$, Maick Willen Fernandes Neves ${ }^{5}$, José Luis Braga de Aquino ${ }^{6}$, Telmo Augusto Barba \\ Belsuzarri ${ }^{7}$, Augusto Ricardo Barba Urena ${ }^{8}$ and Eduardo Augusto Iunes ${ }^{9}$
}

1,2,3 Medicine student from Pontifical Catholic University of Campinas, Brazil

4,7Department of Neurosurgery from Pontifical Catholic University of Campinas, Brazil

${ }^{5}$ Post-Graduation at the State Server Hospital (IAMSPE), Brazil

${ }^{6}$ Post Graduation Department at Pontifical Catholic University of Campinas, Brazil

${ }^{8}$ Cardiologist, Intensive Care Medicine, Pontifical Catholic University of Campinas, Brazil

${ }^{9}$ Professor, Spine Neurosurgereon at Federal University of São Paulo (Unifesp), Brazil

*Corresponding author: Enrico Affonso Barletta, Medicine student from Pontifical Catholic University of Campinas, São Paulo, Barão do Triunfo Street 1052 apartament, Brazil.

Received Date: August 29, 2019

Published Date: September 06, 2019

\section{Abstract}

Background: Glossopharyngeal neuralgia (GPN) is a rare condition typified by paroxysmal episodes of pain along the branches of the glossopharyngeal nerve (CN IX). Its incidence is 7:1,000,000 per year and due to its low incidence, the disease is misjudged. This study aims to present the patient clinically and the therapeutic options.

Methods: We performed a literature review using PUBMED, Clinical Key and Google Scholar. We analyzed 29 articles and the data from 6058 patients that presented with GPN.

Results: The sex incidence showed a small variation. The patients are usually older than 50 years old. The most affected side is the left. The neuroimaging plays a key role to the diagnoses allied with the peculiar pain characterization. Often, GPN is associated with trigeminal neuralgia and hemifacial spasm and it's usually associated with vagus symptoms due to their localization. The posterior circulation is the main cause for the disease and the PICA is the main artery related to the GPN. Comparing Microvascular Decompression (MVD) and Gamma Knife surgery (GKS), we concluded that MVD showed better initial outcomes than GKS, once MVD presented an Odds Ratio (O.R.) of 7,2; it increases in 46,4\% the chance to present an initial good outcome and those values were statistically relevant. Comparing the surgical retreatment ratio and the use of medication after the procedures we also can see better results of MVD.

Conclusion: MVD and GKS are important surgical procedures for this disease. However, MVD showed better results, especially when it comes to initial good outcomes.

Keywords: Glossopharyngeal; Neuralgia; Treatment; Causes; MVD and GKS

\section{Introduction}

Anatomical knowledge is the key point to comprehend correctly the interrelationship between the commitment of the glossopharyngeal nerve (CN IX) and the clinical manifestations. Lack of taste sensation at the posterior third of the tongue, an impaired sensibility of the pharyngeal region, swallowing difficulties, a deviation of the uvula to the healthy side, as well as parotid gland disturbances of secretion are some of the observed changes. The CN IX leave the brainstem through the retro-olivary sulcus and passes the cranial base through the jugular foramen. Inside the foramen it is located the superior ganglion, the smaller of two ganglia, followed by the inferior ganglion. Out of the cranium, the NC IX travels a tortuous path caudally between the internal carotid artery and the internal jugular vein, bending forward and between the stylopharyngeus and styloglossus muscles, innervates the root of the tongue. NC IX emits several branches as the tympanic nerve, that projects itself to the tympanic cavity, where divides into the intramucosal tympanic plexus and the lesser petrosal nerve that 
runs parallel to the greater petrosal nerve and passes through the foramen lacerum to come to the optic ganglion. Besides that, there are parasympathetic secretory fibers directed at the parotid gland when passing through this ganglion [1-3]. The lingual branches contain gustatory fibers for the posterior third of the tongue, that conduct taste sensations to the nuclei of solitary tract, and tonsillar branches supply sensory fibers to the palatine tonsil. Other branches are the muscular branches, to stylopharyngeus muscle, and the pharyngeal branches, to the superior pharyngeal constrictor, palatoglossus and palatopharyngeus muscle. Together with the sympathetic trunk and the vagus nerve $[\mathrm{X}]$ innervates the following muscles: inferior pharyngeal constrictor, tensor veli palatini and uvula $[2,3]$. Sensory fibers to the pharyngeal mucosa and to the pharyngeal glands are CN IX branches too. Still, mechanoreceptors and chemoreceptors at the carotid sinus and carotid body connect to the brainstem with carotid branches, transmitting sensory input, which consists of fibers that are involved in neurovegetative manifestations. The brainstem integrates this sensory afferent signal and modulates the frequency of breathing and the central blood pressure, reflexively. Finally, muscles of the soft palate receive motor fibers of the NC IX, derived from the nucleus ambiguous [2].

Glossopharyngeal neuralgia (GPN) is a rare condition. Its incidence is 7:1,000,000 per year (5 and 9 in women and in men, respectively) and only represents $0.2-1.3 \%$ of the facial pain syndromes $[4,5,6]$. Due to its low incidence, GPN is often misjudged.
It amounts for only 1 in 70 to 1 in 100 of the incidence Trigeminal neuralgia (TN) [7]. Katusic et al. [4] found that the pain location was on the left side in $58 \%$ of cases, on the right side in $17 \%$ and on both sides in $25 \%$. The disease, also, was more prevalent in patients over 50 years $(57 \%)$ [4].

GPN is mostly unilateral syndrome typified by paroxysmal episodes of pain along the auricular and pharyngeal branches of the CN IX and it's often caused by the compression of the CN IX. Neurovascular conflict of the nerve root entry zone or midcistern portion causes demyelination and afferent hyperexcitability of the cranial nerves IX and/or X [8,9]. The pain is well characterized as paroxysmal, of the electrical shooting type, and most times caused by stimulation of the pharynx or deep throat [10]. Patients can experience 30 to 40 episodes of pain daily, usually persisting for less than seconds to 2 minutes. The pain is triggered by chewing, swallowing, or talking. When GPN affects the parasympathetic functions of the vagus nerve patients also can have syncopal episodes, bradycardia, asystole and convulsion [11].

GPN is usually idiopathic3. The most frequent causes are the ones that have an extrinsic compression compound, like neurovascular conflict due to excessive tortuosity or an ectasia of the vertebrobasilar trunk, being a congenital cause, which can be seen in Figure 1. The GPN can also be caused by atherosclerotic phenomena, mainly in elderly people [3].

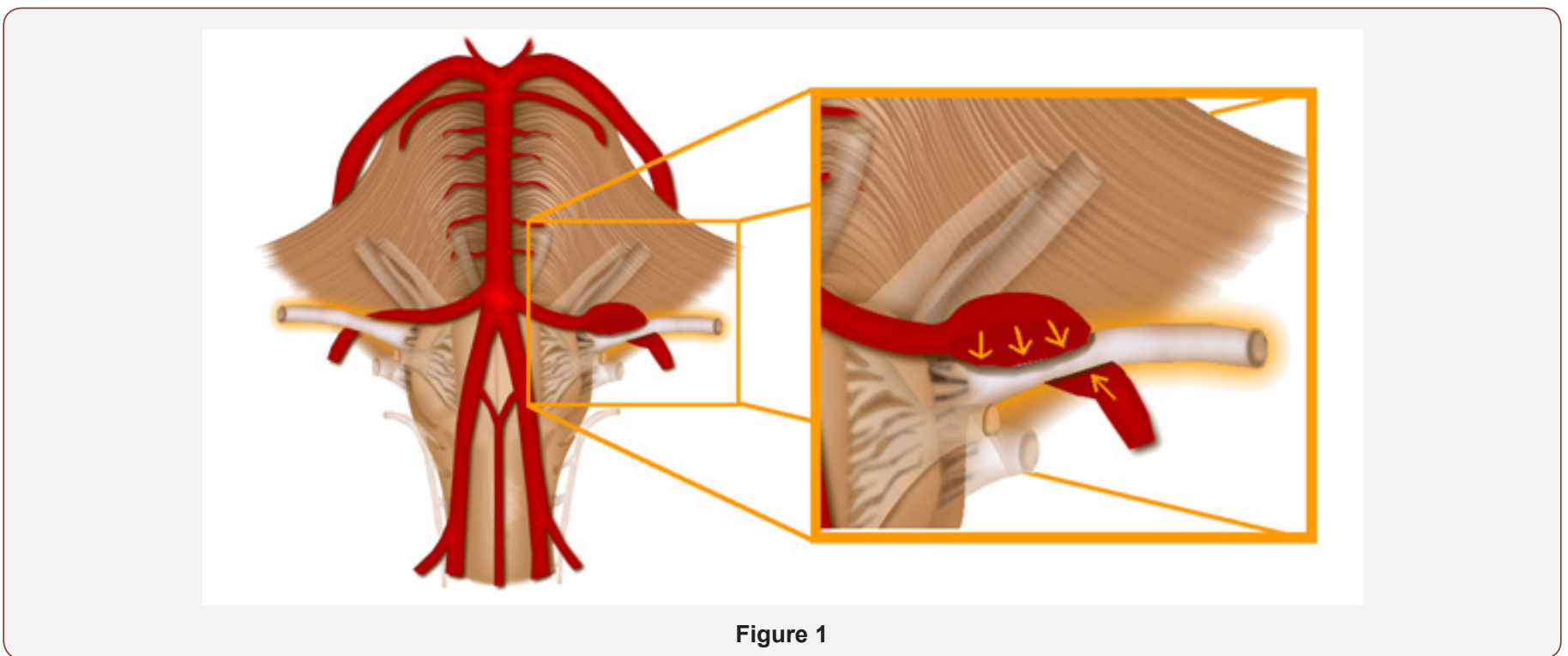

The mechanical compression induces a functional hyperactivity of paroxysmal excitement of the glossopharyngeal nerve and its branches. Secondly, the inflammatory diseases, infectious diseases and demyelinating diseases such as sarcoidosis, HIV and sclerosis plaques [3]. Other causes of secondary GPN include metastasis to petrous temporal bone from breast carcinoma, post-tonsillectomy, carcinoma of the parapharyngeal space carcinoma of the pharynx and nasopharyngeal carcinoma [12]. In addition, besides the compression by arteries and veins, the most common secondary cause of GPN is the Eagle's syndrome, which is the hyperexcitability syndrome, caused by compression of the nerve against an elongated or fractured styloid process or a calcified stylohyoid ligament [12]. Patients that suffered a mandibular trauma may often present with clinical features that may be unrelated to the event itself. The GPN is one of various symptoms, non-related to the trauma that can manifested. The meticulous examination of these patients demonstrated concomitant fractures of the styloid process. The styloid process regards close anatomic relation with vital structures, like the internal carotid artery, internal jugular vein, and cranial nerves such as the glossopharyngeal, that feature justify the structure clinical importance [13], what can be seen in Figure 2. 


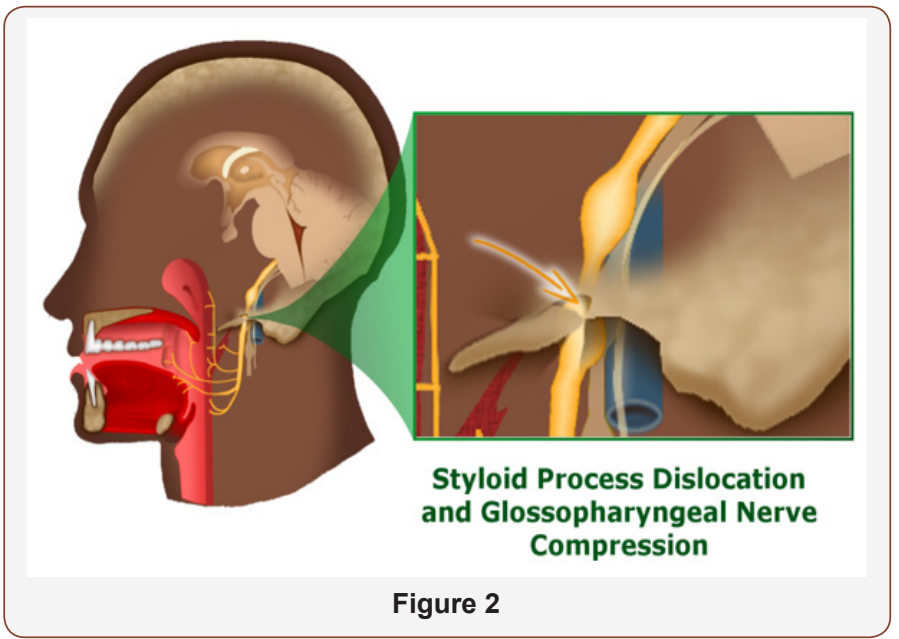

GPN is divided into classic and symptomatic using the International Headache Society diagnosis guidelines witch takes into consideration the durations lasting from fractions of second up to 2 minutes and involving one or more regions of the NC IX, characteristics of the pain as intense, sharp, superficial, stabbing, and/or precipitated by trigger areas or trigger factors and stereotyped in the individual patient, in which the latter requires a causative lesion to be demonstrated by surgery and/or special investigation [14,15,11]. No distal neurological deficits should be observed. The symptomatic class may have a lasting pain that persists between paroxysms and can be associated with sensory damage within the distribution of the nerve [9,3]. The pain experienced in the trigeminal neuralgia is similar to the pain of the GPN, the painful attacks may also be associated with hemodynamic instability that can lead to syncopal episodes [16].

Magnetic resonance imaging (MRI) is an important part for the initial screening in GPN cases and for preoperative imaging assessment to localize the probable cause, such as offending vessels, their course and contact points, also used to analyze the situation of the CN IX $[16,17]$, however the absence of an MRI-identified neurovascular etiology for the GPN should not exclude patients with intractable pain from open surgery [16]. MRI images with three-dimensional data acquisition (3D-MRI) are recommended and can be helpful to diagnose a GPN. In this sense, 3 points are used to identify the CN IX in the exam, being related to the nerve anatomy. Identification of the proximal side of the CN IX is ahead of the inferior part of the cochlear nerve root, a single nerve root of the nerve originates from the brain stem; a single fiber of the $\mathrm{CN}$ IX with a diameter of $<1 \mathrm{~mm}$ and a single fiber of it is located in the anterior part of the jugular foramen [17].

The treatment can be conservative or surgical. Although spontaneous remissions are common, the relapses may be concomitant to drug therapy [8]. Generally, the first approach is the pharmacological, using drugs capable of raising the threshold of nervous excitability, such as neuroleptic, muscle relaxant, and anticonvulsant medications, administered alone or in conjunction, including carbamazepine or oxcarbazepine, gabapentin, pregabalin, and phenytoin. The conservative treatment is recommended for a period of at least 6 months [3]. The interventional therapies are divided as destructive, like the radiosurgery and percutaneous ablative techniques or nondestructive such as the microvascular decompression (MVD) [16,9]. In cases that the conservative therapy proves to be ineffective or when the cause of neuralgia can be easily and safely removed with neurosurgery, such as percutaneous thermal ablation, percutaneous radiofrequency, chemical rhizolysis, ganglionic balloon compression, stereotactic radiosurgery and neurosurgical MVD; the surgical procedures should be considered [3]. Interventional therapies were shown to be effective including MVD and rhizotomy. MVD is one of the most important surgical techniques for the treatment of cranial nerve irritating syndromes [16]. MVD has shown in several studies to have long-dated pain-free outcomes, $80 \%-90 \%$ of subjects treated with a low rate of complications such dysphagia or hoarseness $[10,16]$. MVD also can afford complete relief of pain [16,18]. Other treatment options including radiofrequency ablation and Gamma Knife ablation shown good results [9]. Gamma Knife surgery (GKS) is a minimally invasive and widely recognized as an effective treatment for intractable. Complete pain relief was achieved initially after all interventions [19].

\section{Methods}

We performed a literature review using PUBMED, Clinical Key and Google Scholar. We used glossopharyngeal, neuralgia, treatment, causes, MVD and GKS as our key words. The search was limited to the studies published in English, from 1945 to 2017. We raised a total of 53 articles, but only 29 had the data that interested us. We analyzed the data from 6058 patients that presented with GPN. We observed the patients features; the clinical presentation, the possible causes the treatment outcome for different treatments. The main data we searched was the initial outcome after MVD and GKS. Due to retrospective design of this literature review, we did not apply for ethics committee approval.

\section{Results}

\section{Clinical presentation and patient features}

Vecchi et al. [3] reported the case of an 83 years old man that presented with intense and piercing pain, which was intermittent and paroxysmal, with a duration varying from a few seconds to a maximum of about $1 \mathrm{~min}$, the pain presents a daily frequency, localized in the deep laterocervical site behind the right jaw angle and at the ipisilateral tongue base. The palpation of the root of the neck, yawning, tussive actions and deglutition are triggers and exacerbations factors for the paroxysms. They first occurred with solid food and then appeared with liquids. Therefore, the patient had a weight loss of approximately 6-7 $\mathrm{kg}$ from the time of the symptoms onset. The paroxysms often arise suddenly without prior notice and they often spontaneously regress [3].

Among the 5 patients treated by Álvarez et al.14, 80\% of them are women and $20 \%$ are man. Ferroli et al. [16] analyzed 31 patients that presented with medically intractable GPN. 45\% were man and $55 \%$ were woman; $42 \%$ of them was at the right side and $58 \%$ at the left side. The symptoms had a mean duration of 4.6 years. The compressing vessels were: 71\% the PICA, 22,5\% the VA, 
$3,25 \%$ the AICA, 3,25\% the local veins. The pain was referred to the hemipharynx in $45.2 \%$ of the cases, to the hemipharynx and external ear in 19.3\%, hemipharynx and posterior hemitongue in $12.9 \%$, external ear in $6.5 \%$ and posterior hemitongue in $16.1 \%$ of them. In $16.1 \%$ of the cases GPN was associated with trigeminal neuralgia and in $3.2 \%$ with hemifacial spasm [16].

Rushton et al. [23] analyzed 217 cases of GPN. 57\% of the patients were older than 50 years. $74 \%$ of them presented with spontaneous remissions and $17 \%$ had no periods of pain relief. Involvement was bilateral in $12 \%$ of the cases. The combination of glossopharyngeal and trigeminal neuralgia was seen in 11,5\% of the patients [7]. $40 \%$ of the patients analyzed by Nishihara et al. [17] were man and $60 \%$ were woman; the mean age were 58.4 years. The right side was affected in $30 \%$ of the patients, and the left side was affected in the other $70 \%$ of them [17].

\section{Vascular incidences}

The offending vessels that had been recorded by Nishihara et al. [17] in the operative charts were associated with the posterior inferior cerebellar artery in $90 \%$ of the cases. Posterior inferior cerebellar artery (PICA) was the only affected vessel in $70 \%$ of the patients; a combination of PICA and the vertebral artery (VA) was observed in 10\%, and a combination of PICA and the anterior inferior cerebellar artery (AICA) was observed in 10\%. The offending vessel was AICA alone in $10 \%$ of them. Consistency in the identification of offending vessels by imaging exams was possible in $70 \%$ of the patients. $80 \%$ of the patients with offending vessels attaching to the CN IX at contact points observed on both CISS and 3D-T2R, among those, $87,5 \%$ had a shifted CN IX at the contact points: posteriorly in $43 \%$ of them; superiorly in $14,2 \%$; inferiorly in $14,2 \%$; anteroinferiorly in $14,2 \%$ and posterosuperiorly in $14,2 \%$ [17].

Among the patients treated by Ferroli et al. [16] the compressing vessels were: $71 \%$ the PICA, $22,5 \%$ the VA, 3,25\% the AICA, $3,25 \%$ the local veins. A microvascular compression at the vagoglossopharingeal root entry zone was found in all of the patients during the MVD [16].

\section{Treatments and outcomes}

The study of Panneerselvam et al.18 founded only 1 patient, among 206, presenting with GPN. The patient had a good outcome after being treated with carbamazepine and nerve block [13]. The initial treatment established by Vecchi et al. [3] was conservative, consisting in the pharmacologic administration of pregabalin, starting with low doses of $100 \mathrm{mg} /$ day, followed by a therapeutic increase of $100 \mathrm{mg}$ every 3-4 days, until it reaches the maximum dose of $300 \mathrm{mg} /$ day. The patient presented a good outcome after the treatment, progressively improving the tolerability of paroxysms, which became less frequent until their disappearance. After the third week, the complete resolution of the symptomatology was reached occurred and remained equal for 3 years [3].

Álvarez et al. [20] used GKS to treat GPN in the patients reported. No patients, before GKS, presented any control of the pain with medical management. In the GKS procedure, the nerve can be found using MRI and CT under stereotactic conditions and the target localization was at the level of the glossopharyngeal meatus of the jugular foramen. All the patients that underwent GKS, improved in a period of 3-6 months after the procedure. All of them presented without pain and $60 \%$ of them take no medication after the GKS, but the other $40 \%$ of them continue to take medication. No neurological deficits were observed, after GKS, in none of them [20].

Yomo et al. [19] presented a study involving 2 patients with medically intractable GPN who were treated using GKS, which the target was the distal part of the CN IX. Complete pain relief was achieved initially after all interventions [19]. Isamat et al. [21] percutaneous radiofrequency (RF) thermocoagulation of the inferior petrous ganglion at the jugular foramen has been used to treat 3 patients with essential GPN. $66 \%$ of these patients needed a second RF thermocoagulation. By the end, total of five PR thermocoagulations have been performed and the patients didn't presented significant side-effects and are now pain-free. Deglutition and phonation have remained intact. If it's detected bradycardia and hypotension during the physiological tests, the electrode is misplaced or is spreading current to the vagus nerve [21].

Wang et al. [22] treated 6 patients with coexistent GPN, hemifacial spasm and trigeminal neuralgia. MVD was the treatment of choice for all the patients. Intraoperatively, in all of them, a small posterior fossa was found, which was crowded with cranial nerve roots and cerebellar vessels. To achieve a good outcome is recommended the dissection of the caudal cranial nerves and proximal transposition of the vertebral artery before performing the decompression of the affected nerve roots. Wang et al. [22] also reported that postoperatively the symptom of GPN was relieved immediately in $66 \%$ of the patients and improved with medication in $34 \%$ of them. After and during a 77 months follow-up, no changes, recurrence or any dysfunctions of cranial nerves were observed in none of them. These findings demonstrate how the microvascular decompression may be a good choice for the GPN treatment [22].

Resnick et al. [18] treated 40 patients with MVD of the glossopharyngeal and vagus nerves for the treatment of typical GPN. The study showed that $76 \%$ of the patients attended were painfree and 16\% showed substantial improvement as reported, with a mean follow-up of 48 months. $5 \%$ of the patients died at surgery, duet to hemodynamic lability causing intracranial hemorrhage. $8 \%$ suffered permanent IXth nerve palsy [18].

The patients of Ferroli et al. [16] underwent a MVD. The typical pain of GPN disappeared immediately after surgery in $90.3 \%$ of the cases. In $9.7 \%$ of the patients the pain slowly faded away within the following 2 weeks. $6.4 \%$ of them required repeated surgery due to a drug-resistant recurrence of pain. Between the patients that underwent a repeated surgery an incomplete decompression was found in all of them. $90.3 \%$ of them presented with no pain without medication at long-term follow-up. $9.7 \%$ of the patients required medication, such as a low-dose carbamazepine, to control pain 
paroxysms that reappeared, however the pain was less frequent and less severe than in the preoperative period. No mortality and no long-term surgical morbidity were reported in all cases reported [16].

Nishihara et al. [17] enrolled 10 patients that presented GPN due to neurovascular compression and the treatment of choice was the MVD, after this all the patients achieved symptoms relief [17]. Good relief of pain among the patients treated by Rushton et al. [7] was obtained surgically in only $50 \%$ of the patients after extracranial avulsion of the CN IX or intracranial section at the jugular foramen [7].

Lévêque et al. [23] showed with their study seven patients treated using GKS. The effect was the cure in 5 of 7 patients after 3 months of the surgery and 4 of 7 patients after 7 months of GKS. Four of them had a neurovascular conflict. One patient required repeated surgery because of a recurrence of symptoms. No neurological complications were reported [23].

According to He et al. [24] the 14 patients that were analyzed showing GPN were treated with MVD in the Neurosurgery Department of Lanzhou University Second Hospital between July 2007 and June 2018. With the objective of verifying the risk factors for postoperative delirium (PODE) in patients undergoing MVD, the study shows that one of them presented PODE as an adverse effect to treatment, what shows that the MVD is not free from complications [24].

0'Connor et al. [26] presented in their case-report a woman at the age of 99, suffering from a searing electric shock-like, diagnosed with GPN. Before, she didn't have significant pain relief with medical therapy. The GKS was recommended for her. There were no complications. A substantial pain relief was seen 1 month after the procedure and remained 16 months latter. The patients stayed pain-free and without pain killers [26].

Héroux et al. [26] reports a case of a man, 43-year-old, who felt electric shock-like pain in the left side of the neck, with an 8-year history. MRI demonstrated contact between the CN IX and the left vertebral artery. The patient underwent GKS. Gradual and complete relief of pain was observed a few days after the procedure. 44 months after treatment, the patient remains without medication for pain and did not have pain recurrence [26].

Barletta et al. [27] present in their review data about the initial and the long outcome after a GKS and MVD. They analyzed 2965 patients that underwent MVD and 3093 GKS. They concluded that MVD is more effective than GKS, once this method present higher levels of good outcome [27].

We analyzed these articles to compare initial outcome after MVD and GKS. Therefore, we used the data of Álvarez et al. [20], Yomo et al. [19], Wang et al. [22], Resnick et al. [18], Ferroli et al. [16], Nishihara et al. [17], Lévêque et al. [23], He et al. [24], O'Connor et al. [25], Héroux et al. [26] and Barletta et al. [27]. We analyzed data of 2997 patients that underwent MVD and 3109 that underwent GKS. Comparing MVD with GKS findings regarding good initial outcomes we found statistical relevant values. We calculated the relative risk (R.R), risk difference (R.D), odds ratio (O.R.) and the P-value based on the comparison of MVD group with the GKS group. Our results are showed in Table 1.

Table 1:

\begin{tabular}{|c|c|c|c|c|}
\hline & $\begin{array}{l}\text { Good Initial } \\
\text { Outcome }\end{array}$ & $\begin{array}{l}\text { Bad Initial } \\
\text { Outcome }\end{array}$ & RR: 1.464 & \multirow{3}{*}{$\begin{array}{c}\text { P-Value } \\
<0.0000001\end{array}$} \\
\hline MVD & 2776 & 221 & RD: $29.36 \%$ & \\
\hline GKS & 1967 & 1142 & O.R: 7.293 & \\
\hline
\end{tabular}

We also compared the surgical retreatment ratio and the use of medication after the MVD, GKS. In Graph 1 the surgical retreatment ratio can be seen, data about percutaneous radiofrequency was also presented to show how GKS and MVD are better than other used procedures. The graph shows data of Lévêque et al. [12], Ferroli et al. [16] and Isamat et al. [11] In Graph 2 the percentage of patients who used pain medication after the procedure is shown. This graph presents data of Álvarez et al. [23], Ferroli et al. [16] and Wang et al. [6] (Figure 3 \& 4).

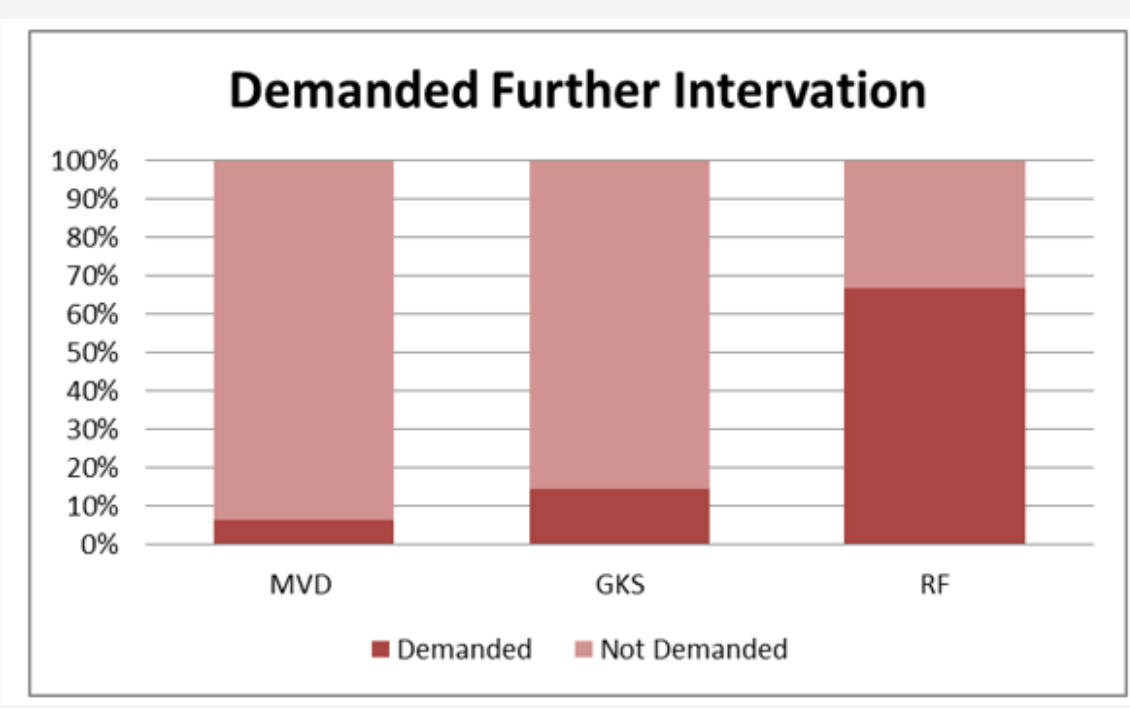

Figure 3: Percentage of patients that required new surgical intervention. 


\section{Percentage of patients using medication after the procedure}

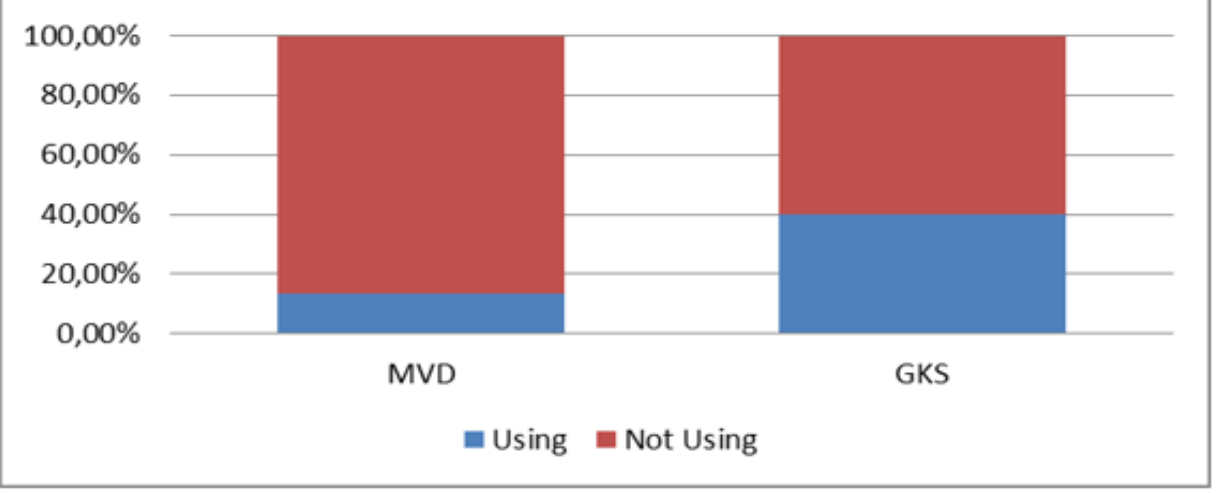

Figure 4: Percentage of patients who used pain medication after the procedure.

\section{Discussion}

Among the patients analyzed, 20 patients were man (42,5\%) and 27 were woman $(57,5 \%)[16,20,17,3]$. The majority of patients are older than 50 years old $[17,7]$. The diagnosis of GPN is clinical, but neuroimaging plays a key role in detecting its causes, such as vascular compression, tumors or demyelinating plaques [16]. The pain is the most important point in the diagnose and it was intermittent and paroxysmal with a short duration in 161 cases (of 218) $[7,3]$. The symptoms were right sided at 17 case and left sided at 25 cases (of 42) [16,17,3]. Many times the GPN is associated with trigeminal neuralgia and hemifacial spasm [16]. The coexistence of symptoms due to an injury at the IXth and Xth cranial nerves is well explained because both share the retroolivar sulcus as their origin, which can then be distorted or compressed by a vessel, for example. Additional evidence comes from the well-known fact that the descending trigeminal tract is shared by pain fibers of the cranial nerves IX and $\mathrm{X}$ as the first relay station [16]. In the present study the GPN was related to trigeminal neuralgia in $12 \%$ of studies with data $[16,7]$. The GPN pain is already well characterized, because its manifestation is localized at the innervation sites of the CN IX. So, the hemipharynx, the external ear and the posterior hemitongue are common sites as it's demonstrated by Ferroli et al. [16] and Vecchi et al. [3].

When the GPN presents a neurovascular genesis, the posterior circulation is the responsible for the CN IX injuries. The main offending vessels were the PICA at $75,6 \%$ of cases, the AICA in $4,8 \%$ and the VA in $19,5 \%$ of 41 cases presented by Ferroli et al. [16] and by Nishihara et al. [17]. Patients that present with GPN, should be treated initially with a pharmacological approach and many times this treatment is enough $[13,3]$. Despite the usage of new anti-epileptic drugs to the treatment of GPN, surgery is still needed in cases of drug intolerance and/or refractoriness [15]. The interventional therapies also must be considered and they are being increasingly used as the first line treatment for the GPN. They are divided as destructive, like the radiosurgery and percutaneous ablative techniques or nondestructive such as the microvascular decompression (MVD) [16,9]. The MVD was used in 2997 patients. The GKS was presented as a good alternative treatment, 3109 patients underwent the procedure in this study. Comparing those procedures, we concluded that MVD showed better initial outcomes than GKS, once MVD presented an 0.R of 7,2; it increases in 46,4\% the chance to present an initial good outcome and those values were statistically relevant. Comparing the surgical retreatment ratio and the use of medication after the procedures we also can see better results of MVD [16,12,23,11,6]. Isamat et al. [21] presented good results using PR in 3 patients, the disadvantage is the high rates of the need to remake the procedure [21].

Most surgical treatments focus at the lesion of the glossopharyngeal and vagal fibers, such as a direct surgical neurotomies or a direct section of glossopharyngeal and vagal nerves in the cerebello-pontine angle [16]. The surgical procedures showed bad results, so they are being each time less used due to its innefectiveness23. Sectioning those nerves often follows a severe and persistent dysphonia and dysphagia, besides that this technique does not necessarily prevent the recurrence of pain. In cases of GPN where a safe and effective treatment such as MVD is available, the usage of injurious treatments is inadequate. The solid study made by Ferroli et al. [16] demonstrates that the MVD is a procedure that always has to be considered for the treatment of GPN. Therefore, MVD should be considered as the first-choice treatment in all cases of drug-resistant GPN, besides that the age of the patient was not a risk factor for the procedure [16].

\section{Conclusion}

The sex incidence showed a small variation. The patients are usually older than 50 years old. The most affected side is the left. The neuroimaging plays a key role to the diagnoses allied with the peculiar pain characterization. Most times has a short duration and it's intermittent affecting the CN IX innervation sites, such as the hemipharynx, the external ear and the posterior hemitongue. Often, GPN is associated with trigeminal neuralgia and hemifacial spasm and it's usually associated with vagus symptoms due to their 
localization. The posterior circulation is the main cause for the disease and the PICA is the main artery related to the GPN. Surgery is still needed in cases of drug intolerance and/or refractoriness. The GKS and other radiofrequency techniques were presented as a good alternative treatment. However, MVD showed better initial outcomes. MVD is a safe and effective treatment for GPN in all age patients. Most patients are able to withdraw from drugs after surgery, so the MVD must be considered as the first choice treatment for patients who are medically intractable and has the procedure indication. Surgical procedures showed bad results, so they are being less used.

\section{Acknowledgement}

None.

\section{Conflict of Interest}

No conflict of interest.

\section{References}

1. Rovenstein EA, Papper EM (1948) Glossopharyngeal nerve block. Am J Surg 75(5): 713-715

2. Sobotta FP (2010) Brain and Spinal Cord Atlas of Human Anatomy 3(12): 211-342.

3. Vecchi M, Pereira Mestre R, Thiekalamuriyil SL, Cartolari R (2017) A Rare Case of Glossopharyngeal Neuralgia due to Neurovascular Conflict. Case Reports in Neurology 9(3): 309-315.

4. Katusic S, Williams DB, Beard M, Bergstralh E, Kurland LT (1991) Incidence and clinical features of glossopharyngeal neuralgia, Rochester, Minnesota 1945-1984. Neuroepidemiology 10: 266-275.

5. Manzoni GC, Torelli P (2005) Epidemiology of typical and atypical craniofacial neuralgias. Neurol Sci 26: 65-67.

6. Sarlani E, Grace EG, Balciunas BA, Schwartz AH (2005) Trigeminal neuralgia in a patient with multiple sclerosisand chronic inflammatory demyelinating polyneuropathy. J Am Dent Assoc 136: 469-476.

7. Rushton JG, Stevens JC, Miller RH (1981) Glossopharyngeal (vagoglossopharyngeal) neuralgia: a study of 217 cases. Arch Neurol 38(4): 201-205.

8. Pearce JMS (2006) Glossopharyngeal Neuralgia. Department of Neurology, Hull Royal Infirmary, Hull, UK. Eur Neurol 55: 49-52.

9. Reddy GD, Viswanathan A (2014) Trigeminal and Glossopharyngeal Neuralgia. Neurol Clin 32: 539-552.

10. Chen J, Sindou M (2015) Vago-glossopharyngeal neuralgia: a literature review of neurosurgical experience. Acta Neurochir (Wien) 157(2): 311-321.

11. Headaches and Other Head Pain Kathleen B. Digre Goldman-Cecil Medicine, 398, 2356-2364.e2 Twenty-Fifth Edition.

12. KBK Soh (1999) The glossopharyngeal nerve, glossopharyngeal neuralgia and the Eagle's syndrome - current concepts and management. Singap Med J 40(10): 659-665.

13. Panneerselvam E, Balasubramaniam S, Sharma AR, Murugadoss P, Chellappazham S, et al. (2018) Fractures of the Stylomandibular Complex: A Prospective Study Proposing a Classification and Clinical
Protocol. J Oral Maxillofac Surg 76(8): 1734-1744.

14. Headache Classification Committee of the International Headache Society (IHS) (2013) The International Classification of Headache Disorders, $3^{\text {rd }}$ edition (beta version). Cephalalgia 33(9): 629-808.

15. Headache Classification Subcommittee of the International Headache Society. The International Classification of Headache Disorders: $2^{\text {nd }}$ edition. Cephalalgia.

16. Ferroli P, Fioravanti A, Schiariti M, Tringali G, Franzini A, et al. (2009) Microvascular decompression for glossopharyngeal neuralgia: a long-term retrospectic review of the Milan-Bologna experience in 31 consecutive cases. Acta Neurochir (Wien) 151(10): 1245-1250.

17. Nishihara M, Noguchi T, Kawashima M, Azama S, Matsushima K, et al. (2017) Magnetic Resonance (MR) Imaging Assessment for Glossopharyngeal Neuralgia: Value of Three-Dimensional T2-Reversed MR Imaging (3D-T2R) in Conjunction with Other Modes of 3D MR Imaging. Polish Journal of Radiology 82: 638-644.

18. Resnick DK, Jannetta PJ, Bissonnette D, Jho HD, Lanzino G (1995) Microvascular decompression for glossopharyngeal neuralgia. Neurosurgery 36: 64-69.

19. Yomo S, Arkha Y, Donnet A, Régis J (2009) Gamma Knife surgery for glossopharyngeal neuralgia. J Neurosurg 110(3): 559-563.

20. Martínez-Álvarez R, Martínez-Moreno N, Kusak ME, Rey-Portolés G (2014) J Glossopharyngeal neuralgia and radiosurgery. Neurosurg 121 Suppl: 222-225.

21. Isamat F, Ferran E, Acebes JJ (1981) Selective percutaneous thermocoagulation rhizotomy in essential glossopharyngeal neuralgia. J Neurosurg 55(4): 575-580.

22. Wang YN, Zhong J, Zhu J, Dou NN, Xia L, Visocchi M, et al. (2014) Microvascular decompression in patients with coexistent trigeminal neuralgia, hemifacial spasm and glossopharyngeal neuralgia. Acta Neurochir (Wien) 156(6): 1167-71.

23. Lévêque M, Park MC, Melhaoul A, Yomo S, Donnet A, et al. (2011) Gamma Knife radiosurgery for glossopharyngeal neuralgia Marseille: experience. J Radiosurg SBRT 1: 41-46.

24. He Z, Cheng H, Wu H, Sun G, Yuan J (2019) Risk factors for postoperative delirium in patients undergoing microvascular decompression. PLoS ONE 14(4): e0215374.

25. O'Connor J K, Bidiwala S (2013) Effectiveness and Safety of Gamma Knife Radiosurgery for Glossopharyngeal Neuralgia, Baylor University Medical Center Proceedings 26(3): 262-264.

26. Héroux F, Mathieu D (2015) Treatment of Glossopharyngeal Neuralgia by Gamma Knife Radiosurgery. Can J Neurol Sci 42: 350-352.

27. Enrico Affonso Barletta, Caique Chagas Cavuto, Ranieri Henrique Moraes Lopes Gaspar, João Flávio Mattos Araújo, Maick Willen Fernandes Neves, et al. (2018) "Treatment of Trigeminal and Glossopharyngeal Neuralgia: A Comparison between the Outcome of the Microvascular Decompression and the Gamma Knife Radiosurgery". EC Neurology 10.7: 600-604.

28. Ross AG, Jivraj I, Rodriguez G, Pistilli M, Chen JJ, et al. (2018) Retrospective, Multicenter Comparison of the Clinical Presentation of Patients Presenting with Diplopia From Giant Cell Arteritis vs Other Causes. Journal of Neuro-Ophthalmology 39(1): 8-13.

29. Pagura JR, Schnapp M, Passarelli P (1983) Percutaneous radiofrequency glossopharyngeal rhizotomy for cancer pain. Appl Neurophysiol 46(14): 154-159. 